\title{
Does Sex in Managerial Positions Really Matter? Differences in Work-Related Feelings and Behaviors
}

\author{
Magdalena Anna Jaworek (D) \\ Tadeusz Marek ${ }^{2}$ \\ Waldemar Karwowski ${ }^{3}$ \\ 'Department of Organizational Behavior, \\ Faculty of Management and Social \\ Communication, Jagiellonian University, \\ Krakow, Poland; ${ }^{2}$ Department of \\ Neurocognitive Science and \\ Neuroergonomics, Jagiellonian University, \\ Faculty of Management and Social \\ Communication, Jagiellonian University, \\ Krakow, Poland; ${ }^{3}$ Department of \\ Industrial Engineering and Management \\ Systems, University of Central Florida, \\ Orlando, FL, USA
}

Correspondence: Magdalena Anna Jaworek

Department of Organizational Behavior, Faculty of Management and Social Communication, Jagiellonian University, Łojasiewicza 4, Kraków, 30-348, Poland Tel +48 I2 6645784

Email magdalena.jaworek@uj.edu.pl
Purpose: The aim of the current study was to test hypotheses regarding differences in workrelated feelings (ie, dejection, anxiety, anger, and happiness) and behaviors (aggressive, avoidance-passive, and proactive) between males and females, managers and nonmanagers, and male and female managers.

Methods: This survey-based study included a total of 3019 respondents, consisting of 502 managers and 2517 employees working in non-managerial positions. Data were collected using two questionnaires developed by the authors: the scale of work-related affective feelings (WORAF) and the scale of work-related behaviors (WORAB).

Results: The results revealed significant differences between managers and non-managers, with managers being happier in their jobs and exhibiting more proactive behaviors. However, there were no differences in work-related feelings or work-related behaviors between males and females in the total sample of respondents or in the group of employees holding managerial positions.

Conclusion: In terms of work-related feelings and behaviors, there are no sex differences among working people. However, some differences between managers and non-managers were observed

Keywords: gender differences, managers, non-managers, work-related affective states, work-related behavior strategies, Bayesian approach

\section{Plain Language Summary}

Regardless of the country or culture, it's mostly men who occupy a majority of managerial positions all over the world. It seems that common sex stereotypes, which lead to believe that males are better managers, are generally responsible for such a situation. These stereotypes can often make it difficult for women to successfully pursue careers in management. Therefore, the objective of the current study is to investigate if the common beliefs regarding men's and women's managerial capabilities are actually justified. In our study, we focused on psychological characteristics such as work-related feelings (including dejection, anxiety, anger, and happiness) and behaviors (aggressive, avoidance-passive, and proactive). A total of 3019 respondents, comprising 502 managers and 2517 employees working in non-managerial positions, participated in the survey-based study. While the results revealed significant differences between managers and non-managers, there were no differences in work-related feelings or work-related behaviors between males and females in the overall group of respondents or in the managerial group. The obtained results are consistent with other similar researches and argue for the implementation of organizational policies based on sex equality.

\section{Introduction}

All occupations require an adequate set of knowledge, skills, abilities, and predispositions. Some of these professional competencies are also commonly associated 
with sex. Therefore, some professions are perceived as masculine or feminine to some extent. Shinar ${ }^{1}$ reported three major criteria for occupational gender-typing. The first criterion is focused on character traits. Assertiveness, a willingness to take risks, and independence were ascribed to men, while sensitivity to other's needs, sympathy, and the ability to soothe hurt feelings were attributed to women. ${ }^{2}$ The second criterion is related to physical capabilities, such as strength and physical endurance for men and delicateness for women. The third criterion for occupational gender-typing is based on the proportion of women to men employed in certain occupations (eg, nursing is dominated by women, while driving positions are dominated by men).

In this regard, management positions are strongly regarded as masculine. ${ }^{3-6}$ Schein's study ${ }^{4}$ and its replications ${ }^{7-9}$ clearly show that managers are perceived as possessing characteristics that are more commonly ascribed to men than to women. Additionally, in one of the most popular questionnaires for evaluating gender roles (Bem Sex Role Inventory), ${ }^{2}$ most items for evaluating masculinity fit the personal traits of the perfect manager (eg, self-reliant, defends own beliefs, leadership ability, acts as a leader, competitive, ambitious, willing to take risks, makes decisions easily, forceful, dominant, etc.).

In addition, manager positions are generally occupied by men worldwide, regardless of the country or culture. For instance, in the European Union, women account for only $36 \%$ of people employed in managerial positions. ${ }^{10}$ In the USA and Australia, women represent almost $40 \%$ of all managers. ${ }^{11,12}$ In Poland, the percentage of women in managerial positions is higher (47\%), corresponding to the third highest rate in the European Union. However, this percentage decreases to $21 \%$ (average of $27 \%$ for $\mathrm{EU}$ members) for board members and 13\% (average of $17 \%$ for EU members) for senior executives. ${ }^{10}$

The disproportions between men and women are generally explained by gender stereotypes and common beliefs that men, because of their predispositions, provide a better fit for managerial positions. ${ }^{8}$ However, gender stereotypes, by generating negative performance expectations (descriptive stereotypes) and creating normative standards (prescriptive stereotypes), can result in limitations to career advancement for both men and women. ${ }^{13}$ As Hyde points out, research on gender differences and similarities are needed to evaluate if gender stereotypes are even accurate, and in the case of a lack of confirmation, to implement appropriate policies. ${ }^{14}$ With regard to sex differences in emotions, cognitive processing, and behaviors among managers, very few studies have examined the extent to which men and women in leadership (...) positions indeed differ on (...) psychological characteristics [p. 221]. ${ }^{15}$ Thus, the aim of the current research was to fill this gap and to examine differences between groups of male and female managers and non-managers with respect to psychological characteristics such as work-related feelings and behaviors.

\section{Literature Review and Hypotheses Work-Related Feelings and Behaviors}

This study considered four emotions, which are commonly regarded as basic: fear, anger, dejection (as derived from sadness), and happiness. ${ }^{16,17}$ These four emotions were chosen because of their relationships with workassociated theoretical constructs. Feelings of anxiety are closely linked to stress as one of the most serious problems among contemporary employees. ${ }^{18}$ Prolonged job stress may result in burnout syndrome ${ }^{19}$ and depressive disorders, ${ }^{20-24}$ which are related to emotions such as anxiety or anger. ${ }^{20,21,25-27}$ In the context of organizational environments, anger is a unique emotion caused by an inequity of power, conflicting interests among superiors, subordinates, and clients, and competition with others for positions and better work conditions. According to some studies, feelings of anger and anxiety, as well as aggressive behaviors, are related to workaholism. ${ }^{28,29}$ Positive emotions, including happiness, are associated with positive work-related constructs such as job satisfaction or work engagement. $^{30-32}$

Emotional states are strictly associated with behaviors that are regarded by psychologists as coping strategies or styles. According to the classical concept developed by Lazarus and Folkman, ${ }^{33}$ coping strategies can be divided into two basic categories: problem-focused (eg, planful problem solving) and emotion-focused (eg, distancing, escape-avoidance). Some academics regard coping methods as a style and add the term "avoidance-oriented" to task- and emotion-oriented ways of coping, denoting these strategies as distinct approaches. ${ }^{34}$ In this study, we focus on work-related behavioral reactions, which are congruent with previously studied work-related affective states, ${ }^{16,17,35}$ ie, proactive behaviors (relating to problemfocused coping) as associated with positive feelings, avoidance behaviors (relating to avoidance coping) as 
related to anxious feelings, passive behaviors (relating to emotion-focused coping) as related to feelings of dejection, and aggressive behaviors as a result of angry feelings. In general, aggressive behaviors are not considered as a distinct coping strategy; instead, they are classified as emotion-focused behaviors. Aggressiveness was included in the current study because of its importance in organizational settings, its role in managerial positions, and potential differences between men and women.

Summarizing, affective states and behaviors are associated with more complicated work-related constructs, such as job satisfaction, work engagement, professional burnout, workaholism, job stress, etc., which influence occupational wellness ${ }^{29,30}$ and have organizational consequences. ${ }^{18}$ Therefore, it is worth investigating antecedents and consequences of work-related feelings and behaviors and their relations to other factors (eg, sex or job position), which helps more accurately understand their nature and improve functioning of the individual in a workplace. Regarding work-related feelings, we used the discrete emotions approach recommended by other academics, ${ }^{36}$ which seemed more useful than a dimensional perspective while investigating the role of emotions in the workplace. ${ }^{37}$

For the purposes of the current research, a scale was developed to measure work-related behavioral reactions. However, statistical analyses revealed only three types of work-related behaviors: proactive, avoidance-passive, and aggressive. Therefore, this taxonomy of behaviors was applied in the current study to verify the proposed hypotheses.

\section{Sex Differences in Terms of Emotions and Behaviors}

It is commonly believed that men and women differ in their experience and expression of emotions. In general, women are perceived as more emotional than men, regardless of the culture. $^{38,39}$ However, few studies have verified these assumptions. These few works have indicated that compared with males, females more often report negative feelings such as sadness or fear. ${ }^{40}$ Additionally, women report depression and emotions of sadness as more intense and long-lasting. ${ }^{41}$ As sadness is combined with passivity ${ }^{17}$ and anxiety is associated with avoidance reactions, ${ }^{16,35}$ it can be assumed that women demonstrate passive and avoidance behaviors to a greater extent than men. Studies have also confirmed that women tend to more frequently use emotion-focused coping (including avoidance), while men more often employ problem-focused coping: $;^{34,42,43}$ however, the differences are very small. For sex differences concerning anger, study results are inconsistent, and such differences between men and women, if they occur, seem to depend on the situation. ${ }^{44}$ However, many findings indicate that men and women do not differ in their reported frequency or intensity of anger, ${ }^{40,41,44-46 ;}$;hich appears to be contrary to the common belief that men are the more aggressive gender. Yet, regarding behaviors arising from affective feelings, some studies have confirmed sex differences in the expression of anger: men express this emotion more behaviorally, whereas women express it verbally. ${ }^{40,44}$ Focusing on positive feelings, a study by Simon and Nath ${ }^{40}$ indicated that, compared with women, men more frequently report emotions such as calmness, being at ease, excitement, being overjoyed, and proud. However, no differences due to gender were found for feelings of contentment and happiness.

In summary, research findings have demonstrated some differences in emotional reactions and expressions between males and females. Yet, it must be stressed that all of these studies have focused on everyday life, without distinctions between different areas. The current study is focused only on work-related affective states and behaviors. Thus, the results may not necessarily overlap with findings from studies regarding emotions and behaviors in general. Nevertheless, due to a lack of research on the studied subject in an organizational context, we formulated hypotheses based on conclusions derived from previous studies concerning general sex differences in feelings and behavioral reactions:

H1: It is assumed there are differences between working men and women in experienced work-related feelings of anxiety, dejection, and happiness, as well as aggressive, avoidance-passive, and proactive behavior.

$\mathrm{H} 2$ : It is predicted that work-related feelings of anger do not differ between males and females.

\section{Differences Between Managers and Non-Managers}

Because specific and often very high requirements are necessary for a potential candidate to be selected for a managerial position, not just anyone can be a manager. Thus, it is obvious that employees holding managerial positions differ from non-managers in terms of professional qualities. A large part of this disparity is related to psychological characteristics. In general, it can be assumed 
that as individuals who must face many difficult situations and make responsible decisions, managers demonstrate higher levels of intra- and interpersonal skills, which are associated with affective states and behaviors (see emotional intelligence construct ${ }^{47}$ ). This assumption is in accordance with Schneider's attraction-selection-attrition theory. ${ }^{48}$ Schneider states that attraction to the organization, selection by it, and attrition from it yield particular kinds of persons in an organization (p. 441). His concept relates to organizations, but it can be applied to occupations as well. As he writes further: similar kinds of people (in this case, managers) are likely to have similar kinds of personalities, are likely to choose to do similar kinds of things, and are likely to behave in similar kinds of ways (p. 441). Work-related feelings of happiness and constructive behaviors may be regarded as signs of professional adaptation, while feelings of anxiety, dejection, and avoidance-passive behaviors denote the opposite. Thus, it can be assumed that managers, as a selected group of employees exhibiting high qualities, demonstrate a more functional profile of work-related emotions and behaviors than nonmanagers.This assumption regarding differences in workrelated feelings between managers and non-managers is also in line with Kemper's power-status theory of emotions. ${ }^{49}$ According to Kemper, affective states primarily depend on one's status and power. An increase in one's status and power, which are related to a managerial position, makes him or her feel more satisfied, happy, contented, safe, and secure. However, situations in which another's power is excessive (or one's own power is insufficient), as in the case of subordinates such as nonmanagers, may be associated with emotions of fear and anxiety. Thus, the following hypothesis was formulated:

H3: In comparison with non-managers, managers demonstrate lower levels of work-related feelings of dejection and anxiety and avoidance-passive behaviors, with higher levels of work-related feelings of happiness and constructive behaviors.

For feelings of anger and aggressive behaviors in relation to managerial positions, formulating a hypothesis is more complicated. Unlike sadness and fear, anger is regarded as a powerful emotion, ${ }^{45}$ and those who have power can allow themselves to express their emotions more freely. Thus, it could be expected that people holding managerial positions experience feelings of anger more often and/or more intensely and may demonstrate more aggressive behaviors than non-managers. In contrast, aggressiveness can sometimes be dysfunctional in organizational settings and poorly perceived by subordinates as an inability to control one's own emotions. Moreover, managers are a selected group in terms of the psychological qualities they use to cope with difficult and stressful situations. Therefore, in the face of contradictory premises, we did not formulate a hypothesis about differences in work-related feelings of anger and aggressive behaviors between managers and non-managers.

\section{Differences Between Male and Female Managers}

Although some believe that different leadership styles depend on gender, ${ }^{50}$ very few studies have examined the differences between men and women in terms of professional qualities in managerial positions. Some of these studies do not confirm these assumptions, ${ }^{51}$ similar to studies on psychological characteristics. An extensive study by Wille et $\mathrm{al}^{15}$ showed that both male and female executives demonstrate similar personality traits. Furthermore, it was found that these differences were smaller in a group of managers than for non-managers. No greater sex differences were observed among Pakistani managers regarding emotional intelligence, creative potential, or job satisfaction in a study by Ahmad \& Zadeh. ${ }^{52}$ However, women reported higher levels of managing their own emotions and openness to change. Studies have also shown that female and male leaders do not differ in task style (task or interpersonally oriented) or job effectiveness; yet, some differences in leadership style have been observed. ${ }^{53-55}$ Nonetheless, managerial positions are held by people with high intra- and interpersonal skills and, all above, managerial predispositions, by which these people are selected. Thus, managers form a rather homogenous group in terms of professional competencies. Sex stereotypes can be a barrier for women, inhibiting them from gaining managerial positions. However, those women who succeed must demonstrate at least the same competencies as men, although these competencies are mostly regarded as masculine rather than feminine. ${ }^{15}$ Regarding emotions and behaviors, which can be more complicated entities, the authors did not find any studies examining differences between male and female managers. Thus, based on studies confirming a lack of sex differences in psychological characteristics among managers, the following hypothesis is put forth: 
H4: It is assumed that work-related feelings (anxiety, dejection, anger, and happiness) and behaviors (aggressive, passive-avoidance, and proactive) do not differ between men and women in managerial positions.

\section{Materials and Methods}

Calculations for sample characteristics, correlation analyses, and statistical features of the measurements were performed with IBM SPSS Statistics 24 and AMOS 24. Hypothesis testing was conducted using the R 3.6.2 statistical environment.

\section{Sample}

The sample consists of 3019 occupationally diverse Polish employees, including 63.6\% women. Approximately 29\% were less than 30 years old, almost $34 \%$ were $31-40$ years old, 22\% were $41-50$ years old, approximately $12 \%$ were 51-60 years old, and 2\% were aged over 60 years. The major occupational groups included in the sample were teachers $(19.3 \%)$, nurses $(11.6 \%)$, and managers $(10.6 \%)$. The majority of the sample had been employed at their current work place for 3-5 years $(20.4 \%)$ and in their current position for $3-5$ years $(23.9 \%)$. The average work experience was 14.8 years $(\mathrm{SD}=10.38)$. Participants worked 41.11 hours per week on average $(\mathrm{SD}=11.59)$. Approximately $17 \%$ of the sample $(\mathrm{n}=$ 503) were employees holding managerial positions, regardless of their occupation. Thus, in addition to managers of commercial organizations, headmasters, head nurses, directors of departments in the public sector, and others were found in that group. For the analysis, assignment to the managerial or non-managerial group was based on the self-reports of the participants.

As there can be confusion regarding the use of the terms of "sex" and "gender," it must be stressed that, in the current study, "sex" refers to the biological aspects of maleness or femaleness. ${ }^{56}$ Assignment to the male or female group was also based on self-reports from the participants.

\section{Research Procedure}

This study was conducted in 2017 and 2018 in two phases. The first phase involved preliminary research to develop and test the measures used in phase two. For the hypothesis testing, only data from phase two were used. In both phases, the data were gathered by students pursuing MA degrees in psychology or management, who were instructed on the research methodology and protocol.
Data for the first phase were collected using the paperand-pencil method, and in the second phase also via the Internet (37\% of subjects). The only constraint while collecting data was the professional activity of the participants. The detailed procedures for this research have been described previously. ${ }^{57}$

\section{Measures}

Work-related feelings were assessed with the scale of work-related affective feelings (WORAF) developed and validated by Jaworek, Marek, and Karwowski. ${ }^{57}$ This scale measures four emotions (anxiety, dejection, anger, and happiness) in association with work settings. The WORAF includes eight items on work-related anxiety (eg, "I am concerned that I won't be able to meet work requirements"), five items on work-related dejection ("Most work-related activities make me feel sad and useless"), four items on work-related anger ("Recently, everything related to my work makes me angry"), and seven items on work-related happiness ("I find my work enjoyable"). Items were scored on a 4-point rating scale, ranging from 1 (almost never) to 4 (almost always).

Due to a lack of adequate tools for measuring coping strategies in an organizational setting, a scale was developed for the needs of the current work: the work-related behaviors scale (WORAB). The procedures used to develop this scale are similar to those used for the WORAF. $^{57}$ First, a pool of items was created by a team of seven experts. Four types of work-related behaviors were distinguished that are congruent with work-related affective states: proactive, avoidant, passive, and aggressive. A pool of 31 items was initially chosen for the measurement instrument and tested during phase one of the study. To verify the theoretical structure, a preliminary exploratory factor analysis using the principal axes method and oblimin (oblique) rotation was conducted. This exploratory analysis revealed six factors. Three factors that consisted of only one or two items loading higher than 0.35 were deleted. Seven items were excluded because they did not reach the threshold of item-total correlations $(<0.35)$ in any of the factors. Another factor analysis (principal axes method with oblimin rotation) revealed a three factor solution. Two of the factors overlapped with the theoretical structure. One factor consisted of five items primarily assigned to work-related avoidance, and three to passive behaviors. To test the factorial validity of the 
measure, a subsequent confirmatory factor analysis was performed during Phase 2 of the study.

The final version of the WORAB consisted of 21 items that assess three types of behaviors: proactive, aggressive, and avoidance-passive. The scale includes seven items on work-related proactive behaviors (eg, "When problems occur, I tend to think straight and clearly"), six items on work-related destructive behaviors (eg, "I sometimes throw papers, documents, or objects, hit the keyboard, etc."), and eight items on work-related avoidance-passive behaviors (eg, "I pretend to be busy so I won't have to work on other tasks"). The items were scored on a 4-point rating scale, ranging from 1 (almost never) to 4 (almost always). Details are shown in Table 1.
To assess the internal consistency of the WORAB subscales, Cronbach's alpha was used. The results showed good internal consistency for all three subscales, ranging from 0.80 to 0.83 . Details are shown in Table 2 .

\section{Results}

Hypothesis testing was conducted using the R 3.6.2 statistical environment. ${ }^{58}$ We used Bayesian regression analyses implemented in the brms package. ${ }^{59,60}$ This Bayesian approach was applied because the distributions of the variables were substantially skewed. This method allows for a flexible choice of response distribution and provides stable parameter estimates, even for complex statistical models. For all analyses, we used skew-normal regression in which the skewness constitutes an additional parameter

Table I Items and Factor Loading of the Measures in Study I (with EFA; n = 297) and Study 2 (with CFA; $n=30$ I9)

\begin{tabular}{|c|c|c|c|c|}
\hline \multirow[t]{3}{*}{ Item Wording } & \multicolumn{3}{|c|}{ Study I* } & \multirow{3}{*}{$\begin{array}{c}\text { Study } \\
2 * * \\
\text { CFA }\end{array}$} \\
\hline & \multicolumn{3}{|c|}{ EFA } & \\
\hline & $\mathbf{I}$ & 2 & 3 & \\
\hline \multicolumn{5}{|l|}{ Work-Related Avoidance-Passive Behaviors } \\
\hline I pretend to be busy so I will not have to work on other tasks & 0.69 & & & 0.74 \\
\hline When difficult situations appear, I tend to act like "I'm not here" & 0.67 & & & 0.65 \\
\hline Recently, I do not make any decisions at work - I wait to see what will happen & 0.61 & & & 0.65 \\
\hline $\begin{array}{l}\text { If I feel that I have nothing to lose, I would rather spend my time in activities such as gossiping; work will not } \\
\text { run away }\end{array}$ & 0.58 & & & 0.58 \\
\hline When I am facing a wall, I do not care what happens next & 0.53 & & & 0.67 \\
\hline I do not fight the setbacks that occur at work & 0.53 & & & 0.53 \\
\hline I try to avoid problematic situations & 0.47 & & & 0.38 \\
\hline I try to not bring attention to myself in any way & 0.46 & & & 0.44 \\
\hline \multicolumn{5}{|l|}{ Work-Related Proactive Behaviors } \\
\hline When problems occur, I tend to think straight and clearly & & 0.77 & & 0.71 \\
\hline I solve problems effectively and fast & & 0.76 & & 0.61 \\
\hline I am effectively handling all my tasks & & 0.71 & & 0.72 \\
\hline I am capable of overcoming difficulties that I face at work & & 0.63 & & 0.66 \\
\hline When problems occur, I start analyzing them straight away to solve them & & 0.60 & & 0.63 \\
\hline I do not have any problems with communicating or solving problems at work & & 0.58 & & 0.41 \\
\hline Even when under a high workload, I am capable of finding the energy needed for work & & 0.53 & & 0.72 \\
\hline \multicolumn{5}{|l|}{ Work-Related Aggressive Behaviors } \\
\hline I have aggressive thoughts towards my co-workers/supervisors & & & -0.86 & 0.76 \\
\hline Things happening at work make me mad at my co-workers/supervisors & & & -0.70 & 0.74 \\
\hline I sometimes throw papers, documents, or objects, hit the keyboard, etc. & & & -0.70 & 0.69 \\
\hline I say negative things about my co-workers and leadership & & & -0.66 & 0.67 \\
\hline I shout at my employees and/or co-workers & & & -0.52 & 0.63 \\
\hline Although I did not previously swear, I have started swearing at work & & & -0.49 & 0.55 \\
\hline Explained Variance (\%) & 29.11 & 12.40 & 3.91 & - \\
\hline Explained Total Variance (\%) & \multicolumn{3}{|c|}{46.22} & - \\
\hline
\end{tabular}

Notes: *Measure of sampling adequacy: Kaiser-Meyer-Okin (KMO = 0.906); Bartlett's test of sphericity: $\chi^{2}(210)=2582.00$, p < 0.000I; **Goodness-of-fit indexes for the work-related behavior scale: $\chi^{2}=1197.86, d f=186 ; \chi^{2} / d f=6.44 ; \mathrm{CFI}=0.95 ; \mathrm{TLI}=0.94 ; \mathrm{IFI}=0.95 ; \mathrm{RMSEA}=0.04$. 
Table 2 Means, Standard Deviations, Cronbach's Alpha, and Intercorrelations Among Subscales

\begin{tabular}{|l|c|c|c|c|c|c|c|}
\hline Work-Related & $\mathbf{I}$ & $\mathbf{2}$ & $\mathbf{3}$ & $\mathbf{4}$ & $\mathbf{5}$ & $\mathbf{6}$ & $\mathbf{7}$ \\
\hline I.Feelings of happiness & - & & & & & \\
2.Feelings of anxiety & $-0.44^{* *}$ & - & & & & \\
3.Feelings of dejection & $-0.49^{* *}$ & $0.68^{* *}$ & - & & & \\
4.Feelings of anger & $-0.48^{* *}$ & $0.69^{* *}$ & $0.62^{* *}$ & - & & \\
5.Proactive behavior & $0.45^{* *}$ & $-0.30^{* *}$ & $-0.27^{* *}$ & $-0.18^{* *}$ & - & \\
6.Aggressive behavior & $-0.32^{* *}$ & $0.59^{* *}$ & $0.58^{* *}$ & $0.62^{* *}$ & $-0.22^{* *}$ & - & \\
7.Avoidance-passive behavior & $-0.31^{* *}$ & $0.53^{* *}$ & $0.56^{* *}$ & $0.48^{* *}$ & $-0.33^{* *}$ & $0.62^{* *}$ & - \\
I.Cronbach's alpha & 0.88 & 0.88 & 0.80 & 0.80 & 0.81 & 0.83 & 0.80 \\
2.M & 2.67 & 1.75 & 1.60 & 1.87 & 3.00 & 1.56 & 1.75 \\
3.SD & 0.66 & 0.59 & 0.58 & 0.60 & 0.58 & 0.56 & 0.53 \\
\hline
\end{tabular}

Note: $*^{*} p<0.01$.

and thus heavy tails are considered in the model. The adequacy of the model was verified by posterior predictive checks.

In Bayesian statistics, the goal is to estimate the posterior probability distribution of model parameters (eg, regression coefficients) by integrating the likelihood with the prior probability distribution of the parameter values. We used default priors implemented in brms, which are weakly informative and do not exert much influence on the parameter estimates. ${ }^{59}$ Parameter inferences were obtained by summarizing the posterior distribution with the mean and $95 \%$ confidence intervals $(95 \% \mathrm{CI})$. The effect captured by a parameter was considered statistically significant if the corresponding 95\% CI excluded zero. ${ }^{61}$

The analytical procedure was identical for each dependent variable. First, a model with main effects (ME model) was fitted to the data, which included the main effects of gender and occupation coded with sum-to-zero contrasts and age coded as a monotonic predictor. The regression coefficients for the gender and occupation were used to compare men with women, and managers with nonmanagers, respectively. Next, the interaction of gender and occupation was added to the model (INT model) in order to test hypothesis $\mathrm{H} 4$, and the Bayes factor (BF) in favor of the INT model over the ME model was computed to determine the significance of the effect. The results are presented in Tables 3 and 4 with a precision of two decimal points. To evaluate effect sizes, we used Bayesian $R^{2}{ }^{62}$

The brms package utilizes a sampling procedure to approximate posterior distributions implemented in STAN language. ${ }^{63}$ For each reported model, four chains were always run in parallel, with 8000 iterations in total. The first half of the samples constituted a burning period, and every tenth sample was recorded from the remaining samples, resulting in 1600 recorded samples in total. This sampling procedure was efficient, as indicated by a lack of autocorrelations in the chains, good convergence of the chains (ie, R-hats $<1.01$ ), and visual inspection of the chains and posterior distributions. ${ }^{61}$

\section{Work-Related Feelings}

First, we determined whether gender or occupation differentiated responses to work-related feeling variables. We did not find any significant effects for these two factors, as indicated by nonsignificant regression coefficients for gender and occupation and $R^{2}$ values close to zero for both the ME and INT models. Next, work-related feelings for the anger dimension were investigated. Again, we did not observe any statistically significant effects. Similarly, we did not observe any significant effects of gender or occupation on the dimension of work-related feelings of dejection.

For the model with work-related feelings of happiness as a dependent variable only, some small yet significant effects were observed. Managers exhibited slightly higher positive feelings related to work than non-managers. Details are shown in Figure 1.

In addition, a weak yet significant effect was noted for the control predictor age. Older employees tended to be happier at work than younger employees. Details are shown in Table 3.

\section{Work-Related Behaviors}

For the work-related passive-avoidance behavior dimension, we observed a very small yet statistically significant effect of occupation. Non-managers 


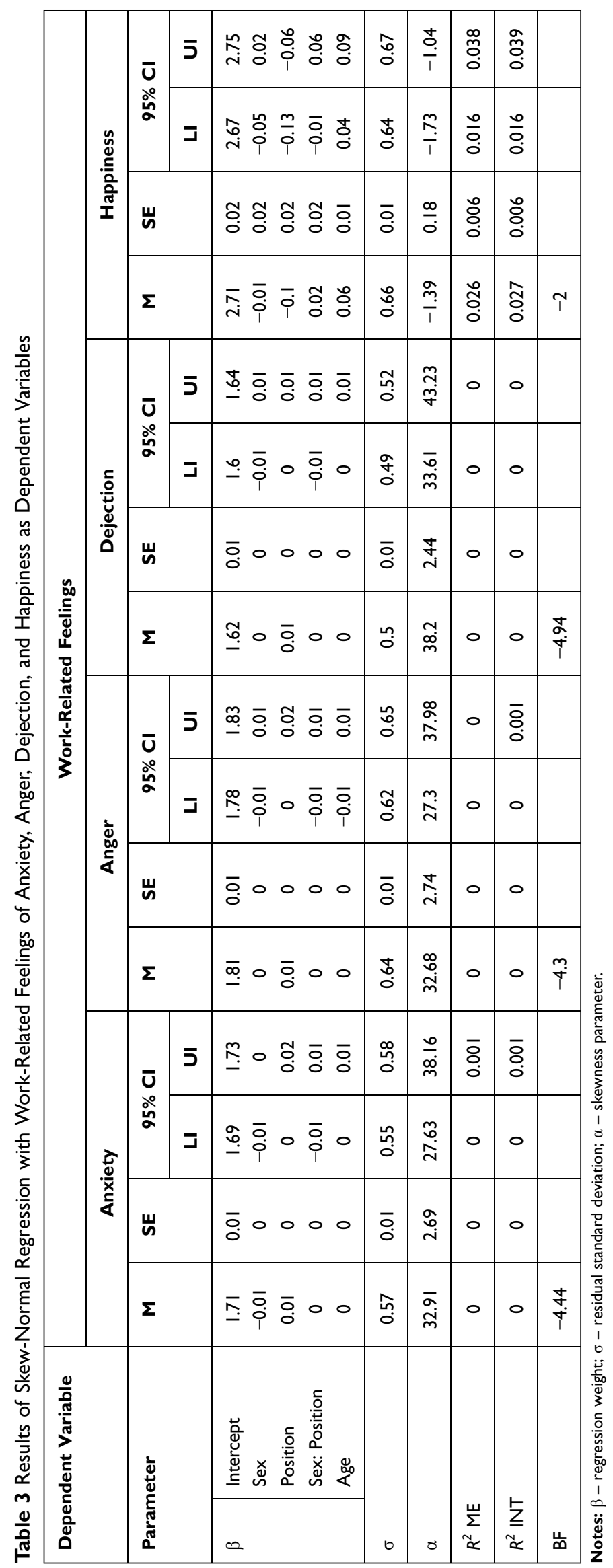


Table 4 Results of Skew-Normal Regression with Work-Related Avoidance-Passive, Proactive, and Aggressive Behaviors as Dependent Variables

\begin{tabular}{|c|c|c|c|c|c|c|c|c|c|c|c|c|c|}
\hline \multirow{2}{*}{\multicolumn{2}{|c|}{ Dependent Variable }} & \multicolumn{12}{|c|}{ Work-Related Behaviors } \\
\hline & & \multicolumn{4}{|c|}{ Avoidance-Passive } & \multicolumn{4}{|c|}{ Proactive } & \multicolumn{4}{|c|}{ Aggressive } \\
\hline & neter & \multirow[t]{2}{*}{$\mathbf{M}$} & \multirow[t]{2}{*}{ SE } & \multicolumn{2}{|c|}{$95 \% \mathrm{Cl}$} & \multirow[t]{2}{*}{$\mathbf{M}$} & \multirow[t]{2}{*}{ SE } & \multicolumn{2}{|c|}{$95 \% \mathrm{Cl}$} & \multirow[t]{2}{*}{$\mathbf{M}$} & \multirow[t]{2}{*}{ SE } & \multicolumn{2}{|c|}{$95 \% \mathrm{Cl}$} \\
\hline & & & & LI & UI & & & LI & UI & & & LI & UI \\
\hline \multirow[t]{5}{*}{$\beta$} & Intercept & 1.66 & 0.01 & 1.64 & 1.69 & 3.08 & 0.02 & 3.05 & 3.11 & 1.57 & 0.01 & 1.56 & 1.59 \\
\hline & Sex & 0 & 0.01 & -0.02 & 0.02 & 0 & 0.01 & -0.03 & 0.03 & 0 & 0 & 0 & 0.01 \\
\hline & Position & 0.07 & 0.01 & 0.05 & 0.09 & -0.13 & 0.01 & -0.16 & -0.11 & 0 & 0 & -0.01 & 0.01 \\
\hline & Sex: Position & 0 & 0.01 & -0.01 & 0.02 & 0 & 0.01 & -0.03 & 0.02 & 0 & 0 & -0.01 & 0.01 \\
\hline & Age & 0.02 & 0.01 & 0 & 0.03 & 0.02 & 0.01 & 0 & 0.04 & 0 & 0 & 0 & 0 \\
\hline \multicolumn{2}{|l|}{$\sigma$} & 0.51 & 0.01 & 0.49 & 0.52 & 0.56 & 0.01 & 0.55 & 0.58 & 0.46 & 0.01 & 0.45 & 0.47 \\
\hline \multicolumn{2}{|l|}{$\alpha$} & 7.43 & 0.77 & 6.12 & 9.12 & -1.79 & 0.16 & -2.12 & -1.47 & 37.96 & 2.42 & 33.32 & 42.71 \\
\hline \multicolumn{2}{|c|}{$R^{2} \mathrm{ME}$} & 0.011 & 0.003 & 0.006 & 0.016 & 0.035 & 0.006 & 0.023 & 0.047 & 0 & 0 & 0 & 0 \\
\hline \multicolumn{2}{|c|}{$R^{2}$ INT } & 0.011 & 0.003 & 0.006 & 0.017 & 0.035 & 0.006 & 0.023 & 0.048 & 0 & 0 & 0 & 0 \\
\hline \multicolumn{2}{|c|}{$\mathrm{BF}$} & -3.76 & & & & -3.36 & & & & -4.95 & & & \\
\hline
\end{tabular}

Notes:s $\beta$ - regression weight; $\sigma$ - residual standard deviation; $\alpha$ - skewness parameter.

exhibited a slightly higher level of work-related avoidance-passive behaviors than managers. Details are shown in Figure 2.

We also observed a weak effect of occupation on the work-related proactive behavior dimension. Managers tended to be more proactive at work than non-managers. (Figure 3).

Finally, we did not observe a significant effect of sex or occupation on work-related aggressive behaviors. Details are shown in Table 4.

\section{Discussion}

The aim of the current study was to verify hypotheses regarding differences in work-related emotions and behaviors between males and females ( $\mathrm{H} 1$ and $\mathrm{H} 2$ ), managers and non-managers (H3), and male and female managers (H4). Hypothesis H1 was not confirmed at all, hypotheses $\mathrm{H} 2$ and $\mathrm{H} 4$ were fully supported, and hypothesis $\mathrm{H} 3$ was partially confirmed.

No differences were observed between women and men in the overall group of working people. These results

\section{WR feelings of happiness}

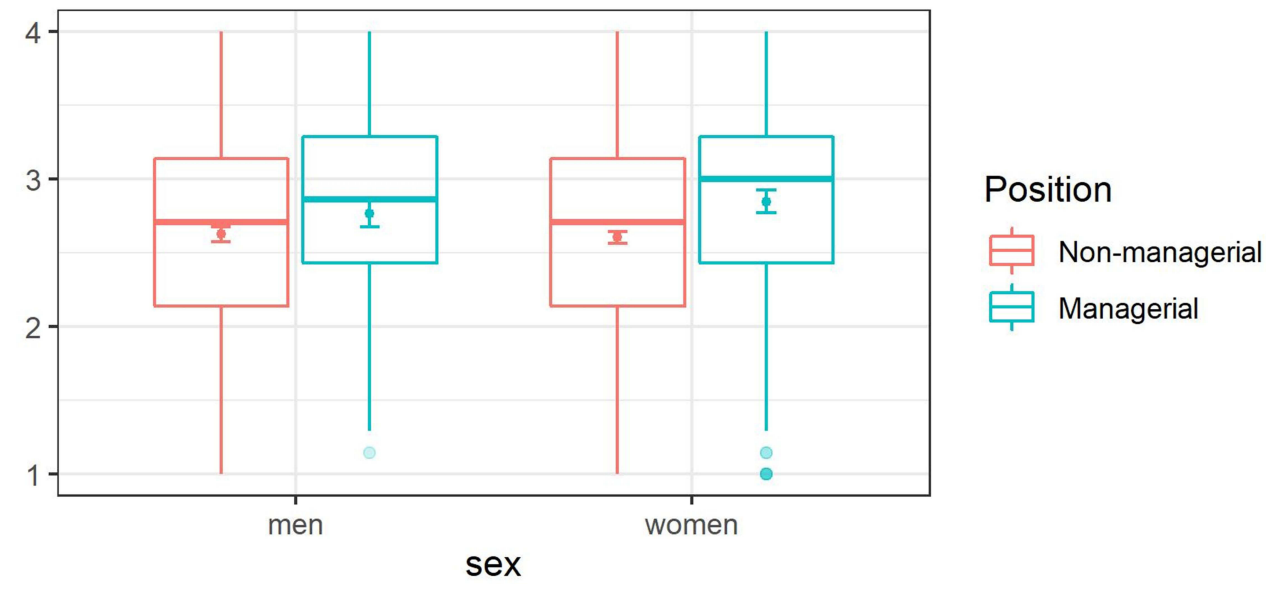

Figure I Effects of sex and occupation on work-related feelings of happiness. The boxplots present the data distribution. The superimposed points with whiskers present estimated marginal means with the $95 \% \mathrm{Cl}$. 
WR passive avoidance behaviors

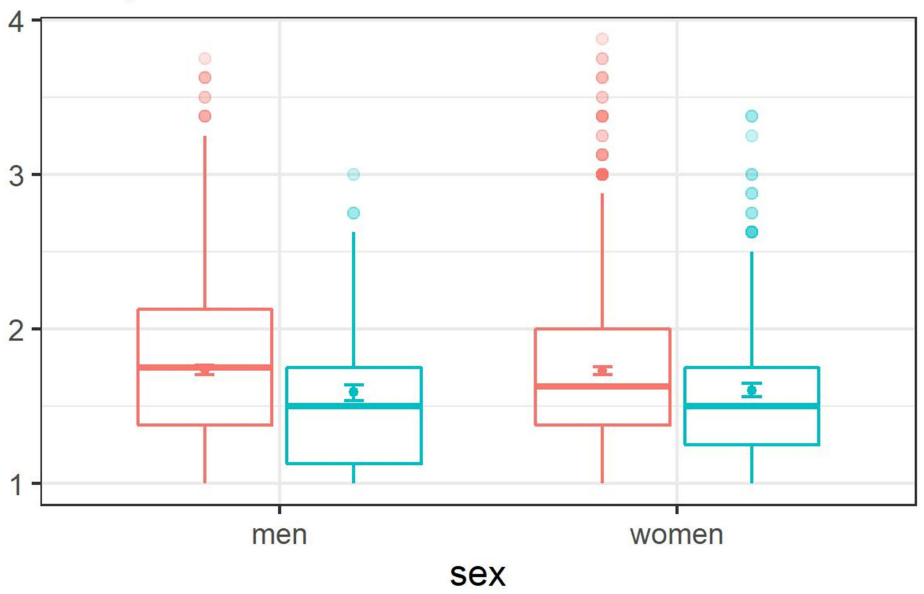

Position

Non-managerial

Managerial

Figure 2 Effects of sex and occupation on work-related passive-avoidance behaviors. The boxplots present the data distribution. The superimposed points with whiskers present estimated marginal means with the $95 \% \mathrm{Cl}$.

\section{WR proactive behaviors}

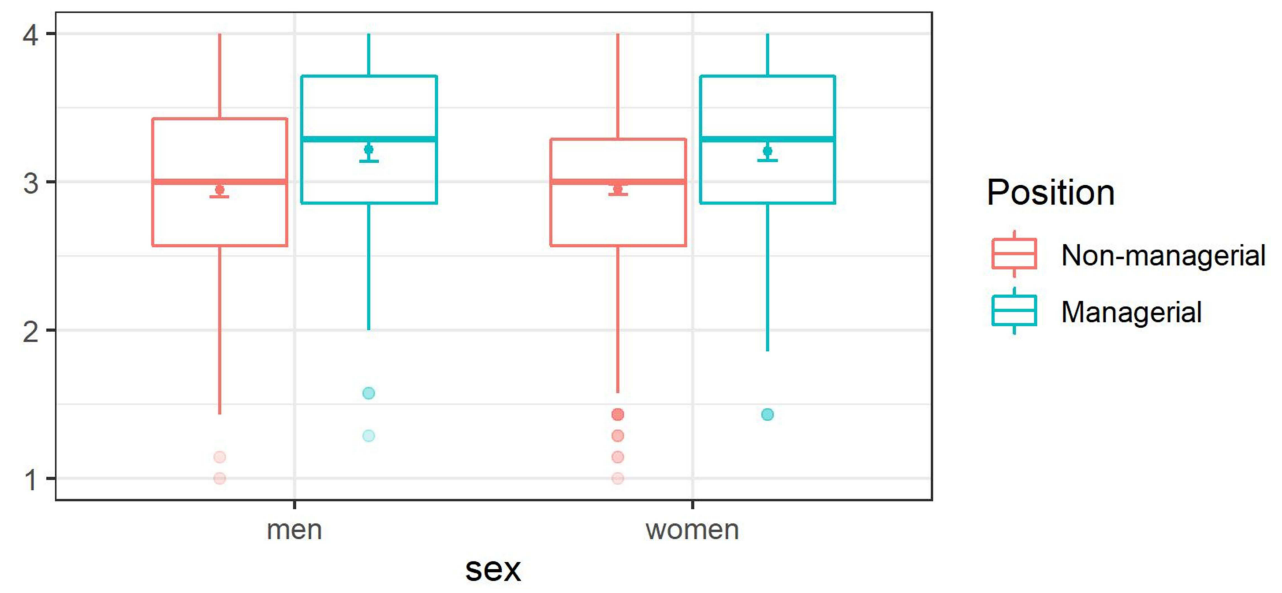

Figure 3 Effects of sex and occupation on work-related proactive behaviors. The boxplots present the data distribution. The superimposed points with whiskers present estimated marginal means with the $95 \% \mathrm{Cl}$.

are partially contrary to those of some studies concerning sex differences in emotions and coping strategies. ${ }^{40}$ However, it must be stressed that our study focuses only on work, not life in general, as previously mentioned. Job environments might similarly affect workers regardless of sex, triggering similar feelings and leading to similar behaviors both among women and men. ${ }^{64-66}$ Yet, work also requires some basic predispositions associated with feelings and behaviors, which may be considered in the recruitment process.

Similarly, no sex differences were observed between the groups of managers and non-managers. These findings may be contrary to common beliefs regarding sex differences among individuals holding managerial positions, but are in accordance with some studies on psychological characteristics. ${ }^{15,52}$ In general, these results show that the selection of employees for managerial positions is influenced by some qualities, which are needed to practice this profession adequately. However, sex does not play a role in this respect. The occupation of manager attracts specific people with certain psychological characteristics, and the best individuals in this regard are chosen in the recruitment and selection process. Thus, managers comprise a selected group of similar people, with sex playing a secondary role here as well as in the overall group of workers. These findings are partially inconsistent with 
Rafaeli and Sutton's theory concerning the role of emotions in organizational life. ${ }^{67}$ According to this theory, sex (the authors used the term gender) as one of the enduring attributes, with societal, occupational and organizational norms, influences the emotions expressed by members of an organization. While the results of the current study concerned felt emotions (not expressed), work-related behaviors (the authors even used the term "emotional behavior") can be regarded as a manifestation and/or a consequence of work-related feelings. Nonetheless, in contrast to the theory outlined by Rafaelli and Sutton, only the role of occupational norms, and not sex, was confirmed.

Differences were noted only between groups of managers and non-managers. Compared to their subordinates, managers demonstrate higher levels of work-related feelings of happiness and proactive behaviors (which may be a consequence of positive affect). These results indicate that experiencing positive emotional states may be a key to achieving occupational success (eg, managerial positions), a conclusion supported by other studies. For example, previous work has shown that managers are more assertive, extraverted, emotionally stable, and optimistic, and display a higher work-drive, agreeableness, customerservice orientation, and conscientiousness, ${ }^{15,68}$ dispositions that can relate to a positive affect. No differences were observed in feelings of dejection, anger, or anxiety or in aggressive behaviors among managers and nonmanagers, which is contrary to part of hypothesis $\mathrm{H} 2$. This finding indicates that employees do not differ in terms of negative affect and aggressiveness at work, regardless of their position in the organization hierarchy. The results also show that managers tend to be less passive and avoidant; however, this effect was very small. The finding of no differences between managers and nonmanagers in negative affect is rather surprising and requires further investigation.

The current results are somewhat congruent with the affective events theory (AET) proposed by Weiss and Cropanzano. ${ }^{69}$ According to this theory, affective reactions leading to work attitudes and affect-driven behaviors depend on work events (as a consequence of work environment features) and dispositions. First, the results of our study showed that work-related feelings are associated with work-related behaviors. With regard to dispositions, the AET proposes that positive and negative affectivity are significant factors influencing job satisfaction and work behaviors. As mentioned above, managers show a higher positive affect compared to representatives of other occupations, which results in increased work-related feelings of happiness. On the other hand, a managerial position, although demanding, is usually associated with power, which relates to more control (ie, work environment features in AET). A combination of high job demands and control, according to Karasek's model, ${ }^{70}$ is a strong predictor of increased motivation and activity (ie, proactive behaviors). As our study did not take into consideration the characteristics of organizational settings and personal traits, these relationships should be investigated in future research.

The obtained results also argument against sex stereotypes. According to these stereotypes, males, due to psychological predispositions, are a better fit for managerial positions. As such stereotypes still exist, ${ }^{71,72}$ our findings argue for the implementation of organizational policies based on sex equality. Such policies would increase the upward potential of employees, minimize feelings of injustice, and positively influence staff morale.

The observed differences in feelings of happiness and proactive behaviors between managers and non-managers are also a starting point for further investigations. These studies should aim to answer the following questions: How does positive affect relate to the management process? Is positive affect important for the social (eg, human resource management), cognitive (eg, decision-making, optimistic thinking) or behavioral (eg, overcoming obstacles, solving practical problems) aspects of a managerial job? Answers to these questions would allow for a better fit between individual managers and specific job tasks. In addition, it will be important to discern the characteristics that contribute to increased positive affect among managers, including such things as the organizational setting, dispositions, and the interactions between them. Identification of these factors would allow emphasis to be placed on these particular features, making those in management positions more effective.

\section{Limitations}

This study has some limitations. Despite the diversity of the sample in terms of occupation, the employment structure of this study does not fully reflect the employment structure in Poland (our study did not include employees in fields such as agriculture or mining). Moreover, this research was conducted only among Polish employees; thus, the results cannot be generalized to other nationalities. Considering the last criterion of gender-typing by 
Shinar ${ }^{1}$ (ie, the proportion of women to men employed in certain occupations, and based on statistics concerning the presence of women in managerial positions), it must be concluded that the position of manager is more or less masculine in some countries than in others. According to Eurostat, ${ }^{10}$ the percentage of women in managerial positions in Poland is $47 \%$, which puts Poland in third place in the European Union, together with Slovenia. In many countries of Europe, women account for less than a third of managers (ie, Cyprus, Germany, Denmark, Italy, Cyprus, Belgium, Austria, or Luxembourg). Such disproportions can reflect cultural norms and attitudes toward male and female roles in society, which may be associated with different results of work-related feelings and behaviors among employees compared with the current study. Thus, in conclusion, further investigations using samples other than Polish employees are recommended.

\section{Conclusions}

In summary, the results of the current study show that, in terms of work-related feelings and behaviors, there are no sex differences among working people. Some differences between managers and non-managers were observed. Thus, considering the first criterion of occupational gender-typing reported by Shinar $^{1}$ (see introduction), the statement that the occupation of manager is definitely masculine is not true, as indicated by the results of this study. Of course, as part of the professional competencies demonstrated by managers, psychological characteristics are not solely manifested as examined feelings and behaviors. However, as other studies have confirmed, there are no differences between male and female managers with respect to psychological characteristics; ${ }^{15}$ rather, these characteristics have a stronger association with occupation than sex. Thus, the idea of think manager - think male ${ }^{73}$ may not reflect reality.

\section{Data Sharing Statement}

The data that support the findings of this study and other materials are available at: https://ruj.uj.edu.pl/xmlui/han $\underline{\text { dle/item } / 282108}$

\section{Ethical Statement}

The current study was carried out with the consent of the Ethics Committee at Jagiellonian University in Krakow, Poland. All subjects provided written informed consent in accordance with the Declaration of Helsinki. Information regarding the confidentiality of the collected data, as well as protecting the subject's identity and assuring their voluntary participation in the study, was also provided.

\section{Disclosure}

The authors report no conflicts of interest in this work.

\section{References}

1. Shinar E. Sexual stereotypes of occupations. J Vocat Behav. 1975;7:99-111. doi:10.1016/0001-8791(75)90037-8

2. Bem SL. The measurement of psychology androgyny. J Consult Clin Psychol. 1974;42(2):155-162. doi:10.1037/h0036215

3. Schein VE. The relationship between sex role stereotypes and requisite management characteristics. J Appl Psychol. 1973;57:95-100. doi: $10.1037 / \mathrm{h} 0037128$

4. Schein VE. The relationship between sex role stereotypes and requisite management characteristics among female managers. $J$ Appl Psychol. 1975;60:340-344. doi:10.1037/h0076637

5. Schein VE. A global look at psychological barriers to women' s progress in management. $J$ Soc Issues. 2001;57(4):675-688. doi:10. $1111 / 0022-4537.00235$

6. Panek PE, Rush MC, Greenawalt JP. Current sex stereotypes of 25 occupations. Psychol Rep. 1977;40(1):212-214. doi:10.2466/pr0.19 77.40.1.212

7. Brenner OC, Tomkiewicz J, Schein VE. The relationship between sex role stereotypes and requisite management characteristics revisited. Acad Manage J. 1989;32(3):662-669. doi:10.2307/256439

8. Heilman ME, Block CJ, Martell RF, And Simon MC. Has anything changed? Current characterizations of men, women, and managers. J Appl Psychol. 1989;74(6):935-942. doi:10.1037/0021-9010.74.6.935

9. Dodge KA, Gilroy FD, Fenzel LM. Requisite management characteristics revisited: two decades later. J Soc Behav Pers. 1995;10 (6):253-264.

10. Eurostat [homepage on the Internet]. 2019. Available from: https://ec. europa.eu/eurostat/documents/portlet_file_entry/2995521/ 3-07032019-BP-EN.pdf/e7f12d4b-facb-4d3b-984f-bfea6b39bb72. Accessed February 22, 2021.

11. Bureau of Labor Statistics BLS [homepage on the Internet]. Women in management; 2017. Available from: https://www.bls.gov/careerout look/2017/data-on-display/women-managers.htm?view_full. Accessed February 23, 2021.

12. Workplace Gender Equality Agency WGEA [homepage on the Internet]. Workforce composition; 2020. Available from: https://data. wgea.gov.au/industries/1\#gender_comp_content. Accessed February 22, 2021.

13. Heilman ME. Gender stereotypes and workplace bias. Res Organ Behav. 2012;32:113-135. doi:10.1016/j.riob.2012.11.003

14. Hyde JS. Gender similarities and differences. Annu Rev Psychol. 2014;65:373-398. doi:10.1146/annurev-psych-010213-115057

15. Wille B, Wiernik BM, Vergauwe J, Vrijdags A, Trbovic N. Personality characteristics of male and female executives: distinct pathways to success? J Vocat Behav. 2018;106:220-235. doi:10.10 16/j.jvb.2018.02.005

16. Plutchik R. The nature of emotions. Am Sci. 2001;89:344-350. doi:10.1511/2001.4.344

17. Oatley K, Johnson-Laird PN. Cognitive approaches to emotions. Trends Cogn Sci. 2014;18(3):134-140. doi:10.1016/j.tics.2013.12.004

18. European Agency for Safety and Health at Work EU-OSHA. [homepage on the Internet]. Calculating the costs of work-related stress and psychosocial risks. European Risk Observatory. Literature Review; 2014. Available from: https://osha.europa.eu/en/publications/calculating-costwork-related-stress-and-psychosocial-risks. Accessed September 4, 2018. 
19. Wu S, Zhu W, Li H, Wang Z, Wang M. Relationship between job burnout and occupational stress among doctors in China. Stress Health. 2007;24(2):143-149. doi:10.1002/smi.1169

20. Ahola K, Honkonen T, Isometsä E, et al. The relationship between job-related burnout and depressive disorders - results from the Finnish Health 2000 Study. J Affect Disord. 2005;88:55-62. doi:10.1016/j.jad.2005.06.004

21. Ahola K, Hakanaen J, Perhoniemi R, Mutanen P. Relationship between burnout and depressive symptoms: a study using the person-centred approach. Burn Res. 2014;1(1):29-37. doi:10.1016/j. burn.2014.03.003

22. Levi L. Working life and mental health - a challenge to psychiatry? World Psychiatry. 2005;4(1):53-57.

23. Rugulies R, Bultmann U, Burr H. Psychosocial work environment and incidence of severe depressive symptoms: prospective findings from a 5-year follow-up of the Danish work environment cohort study. Am J Epidemiol. 2006;163(10):877-887. doi:10.1093/aje/ kwj119

24. Bonde JP. Psychosocial factors at work and risk of depression; a systematic review of the epidemiological evidence. Occup Environ Med. 2008;65(7):438-445. doi:10.1136/oem.2007.038430

25. Fiksenbaum L, Marjanovic Z, Greenglass ER, Coffey S. Emotional exhaustion and state anger in nurses who worked during the sars outbreak: the role of perceived threat and organizational support. Can J Commun Ment Health. 2006;25(2):89-103. doi:10.7870/cjcmh-2006-0015

26. Muscatello MRA, Bruno A, Carioccio C, et al. Association between burnout and anger in oncology versus ophthalmology health care professionals. Psychol Rep. 2006;99:641-650. doi:10.2466/ pr0.99.2.641-650

27. Queiros C, Kaiseler M, da Silva AL. Burnout as predictor of aggressivity among police officers. EJPS. 2013;1(2):110-135.

28. Clark MA, Michel JS, Stevens GW, Howell JW, Scruggs RS. Workaholism, work engagement and work-home outcomes: exploring the mediating role of positive and negative emotions. Stress Health. 2014;30:287-300. doi:10.1002/smi.2511

29. Balducci C, Cecchin M, Fraccaroli F, Schaufeli WB. Exploring the relationship between workaholism and workplace aggressive behaviour: the role of job-related emotion. Pers Individ Dif. 2012;53 (5):629-634. doi:10.1016/j.paid.2012.05.004

30. Van Wijhe CI, Peeters MCW, Schaufeli WB. To stop or not to stop, that's the question: about persistence and mood of workaholics and work engaged employees. Int J Behav Med. 2011;18(4):361-372. doi:10.1007/s12529-011-9143-z

31. Bakker AB, Oerlemans WGM. Subjective well-being in the organization. In: Cameron K, Spreitzer G, editors. The Oxford Handbook of Positive Organizational Scholarship. Oxford University Press; 2011:178-189. doi:10.1093/oxfordhb/9780199734610.013.0014

32. Schaufeli WB, Salanova M. Burnout, boredom and engagement in the workplace. In: Peeters MCW, de Jonge J, Taris TW, editors. An Introduction to Contemporary Work Psychology. New York: John Wiley and Sons; 2013:293-320.

33. Lazarus RS, Folkman S. Stress, Appraisal, and Coping. 1st ed. New York: Springer; 1984.

34. Endler NS, Parker JDA. Multidimensional assessment of coping: a critical evaluation. J Pers Soc Psychol. 1990;58(5):844-854. doi:10.1037//022-3514.58.5.844

35. Izard CE, Ackerman BP. Motivational, organizational, and regulatory functions of discrete emotions. In: Lewis M, Haviland-Jones JM, editors. Handbook of Emotions. New York, London: The Guilford Press; 2000:253-264.

36. Crispim AC, Moraes Cruz R, Baasch D, et al. Measurement of affect: from theoretical and instrumental perspectives. Psychol Res. 2015;5 (2):96-107. doi:10.17265/2159-5542/2015.02.002

37. Ashkanasy MN, Dorris AD. Emotions in the workplace. Annu Rev Organl Psychol Organ Beh. 2017;4:67-90. doi:10.1146/annurevorgpsych-032516-113231
38. Fischer AH, Manstead ASR. The relation between gender and emotion in different cultures. In: Fischer $\mathrm{AH}$, editor. Gender and Emotion: Social Psychological Perspectives. Cambridge: University Press; 2000:71-94. doi:10.1017/CBO9780511628191.005

39. Kring AM, Gordon AH. Sex differences in emotion: expression, experience, and physiology. J Pers Soc Psychol. 1998;74 (3):686-703. doi:10.1037/0022-3514.74.3.686

40. Simon RW, Nath LE. Gender and emotion in the United States: do men and women differ in self-reports of feelings and expressive behavior? Am J Sociol. 2004;109(5):1137-1176. doi:10.1086/382111

41. Brody LR, Hall JA. Gender, emotion, and expression. In: Lewis M, Haviland-Jones JM, editors. Handbook of Emotions. New York: Guilford Press; 2000:338-349.

42. Matud MP. Gender differences in stress and coping styles. Pers Individ Dif. 2004;37:1401-1415. doi:10.1016/j.paid.2004.01.010

43. Meléndez JC, Mayordomo T, Sancho P, Tomás JM. Coping strategies: gender differences and development throughout life span. Span J Psychol. 2012;15(3):1089-1098. doi:10.5209/rev_sjop.2012.v15.n3.39399

44. Kring AM. Gender and anger. In: Fischer AH, editor. Gender and Emotion: Social Psychological Perspectives. New York: Cambridge University Press; 2000:211-231.

45. Fischer A, Rodriguez Mosquera PM, van Vianen AEM, Manstead ASR. Gender and culture differences in emotion. Emotion. 2004;4(1):87-94. doi:10.1037/1528-3542.4.1.87

46. Lucas RE, Gohm CL. Age and sex differences in subjective wellbeing across cultures. In: Diener E, Suh EM, editors. Culture and Subjective Well-Being. The MIT Press; 2000:291-317.

47. Salovey P, Mayer JD. Emotional Intelligence. Imagin Cogn Pers. 1990;9(3):185-211. doi:10.2190/DUGG-P24E-52WK-6CDG

48. Schneider B. The people make the place. Pers Psychol. 1987;40:437-454. doi:10.1111/j.1744-6570.1987.tb00609.x

49. Kemper TD. Power and status and the power-status theory of emotions. In: Stets JE, Turner JH, editors. Handbook of the Sociology of Emotions. Boston: Springer; 2006:87-113. doi:10.1007/978-0-387-30715-2_5

50. Eagly AH. Female leadership advantage and disadvantage: resolving the contradictions. Psychol Women Q. 2007;31(1):1-12. doi:10.1111/ j.1471-6402.2007.00326.x

51. Donnell SM, Hall J. Men and women as managers: asignificant case no significant difference. Organ Dyn. 1980;8(4):60-77. doi:10.1016/ 0090-2616(80)90049-2

52. Ahmad SM, Zadeh ZF. Gender differences on the variable of emotion intelligence, creative-potential and job satisfaction in managers. Pak J Psychol. 2016;47(2):61-77.

53. Eagly AH, Johnson BT. Gender and leadership style: a meta-analysis. Psychol Bull. 1990;108(2):233-256. doi:10.1037/0033-2909.108. 2.233

54. Eagly AH, Karau SJ, Makhijani MG. Gender and the effectiveness of leaders: a meta-analysis. Psychol Bull. 1995;117:125-145. doi:10.1037/0033-2909.117.1.125

55. Eagly AH, Johannesen-Schmidt MC, Van Engen ML. Transformational, transactional, and laissez-faire leadership styles: a meta-analysis comparing women and men. Psychol Bull. 2003;129:569-591. doi:10.1037/0033-2909.129.4.569

56. American Psychological Association. Gender. Available from: https:// dictionary.apa.org/gender. Accessed September 7, 2021.

57. Jaworek M, Marek T, Karwowski W. The scale of work-related affective feelings (WORAF). Appl Ergon. 2020;82:102945. doi:10.1016/j.apergo.2019.102945

58. R Core Team [homepage on the Internet]. R: a language and environment for statistical computing. Vienna: R Foundation for Statistical Computing; 2019. Available from: https:/www.r-project.org/. Accessed December 2, 2021.

59. Bürkner P. brms: an R package for Bayesian multilevel models using stan. J Stat Softw. 2017;80(1):1-28. doi:10.18637/jss.v080.i01

60. Bürkner P. Advanced Bayesian multilevel modeling with the R package brms. $R$ J. 2018;10(1):395-411. doi:10.32614/RJ-2018-017 
61. Kruschke J. Doing Bayesian Data Analysis: A Tutorial with R, JAGS, and Stan. 2nd ed. Academic Press; 2014.

62. Gelman A, Goodrich B, Gabry J, Vehtari A. R-squared for Bayesian regression models. Am Stat. 2019;73(3):307-309. doi:10.1080/ 00031305.2018.1549100

63. Carpenter B, Gelman A, Hoffman MD, et al. Stan: a probabilistic programming language. J Stat Soft. 2017;76(1):1-32. doi:10.18637/ jss.v076.i01

64. Watson J, Robinson S. Adjusting for risk in comparing the performances of male-and female-controlled SMEs. J Bus Ventur. 2003;18 (6):773-788. doi:10.1016/S0883-9026(02)00128-3

65. McCarty WP, Garland BE. Occupational stress and burnout between male and female police officers. Policing. 2008;30(4):672-691. doi:10.1108/13639510710833938

66. Tazekand EF, Nafar N, Keramati R. The relationship between marital satisfaction and job satisfaction among employees of Social Welfare Organization at Tehran Branches. Life Sci J. 2013;10(6):804-812.

67. Rafaeli A, Sutton R. The expression of emotion in organizational life. Res Organ Behav. 1989;11:1-42.

68. Lounsbury JW, Sundstrom ED, Gibson LW, Loveland JM, Drost AW. Core personality traits of managers. J Man Psychol. 2016;31 (2):434-450. doi:10.1108/JMP-03-2014-0092
69. Weiss HM, Cropanzano R. Affective events theory: a theoretical discussion of the structure, causes and consequences of affective experiences at work. In: Staw BM, Cummings LL, editors. Research in Organizational Behavior: An Annual Series of Analytical Essays and Critical Reviews. Greenwich, CT: JAI Press; 1996:1-74.

70. Karasek RA. Job demands, job decision latitude, and mental strain: implications for job redesign. Adm Sci Q. 1979;24(2):285-308. doi: $10.2307 / 2392498$

71. Tabassum N, Nayak BS. Gender stereotypes and their impact on women's career progressions from a managerial perspective. IIM Kozhikode Soc Manag Rev. 2021;10(2):192-208. doi:10.1177/ 2277975220975513

72. Gerdman D. How gender stereotypes kill a woman's self-confidence? Harvard Business School; 2019. Available from: https://hbswk.hbs.edu/ item/how-gender-stereotypes-less-than-br-greater-than-kill-a-womans-less-than-br-greater-than-self-confidence. Accessed September 24, 2021.

73. Schein VE, Mueller R, Lituchy T, Liu J. Think manager-think male: a global phenomenon? J Organ Behav. 1996;17(1):33-41. doi:10.1002/(SICI)1099-1379(199601)17:1<33::AID-JOB778>3.0. $\mathrm{CO} ; 2-\mathrm{F}$
Psychology Research and Behavior Management

\section{Publish your work in this journal}

Psychology Research and Behavior Management is an international, peer-reviewed, open access journal focusing on the science of psychology and its application in behavior management to develop improved outcomes in the clinical, educational, sports and business arenas. Specific topics covered in the journal include: Neuroscience, memory and decision making; Behavior modification and management; Clinical

\section{Dovepress}

applications; Business and sports performance management; Social and developmental studies; Animal studies. The manuscript management system is completely online and includes a very quick and fair peer-review system, which is all easy to use. Visit http://www. dovepress.com/testimonials.php to read real quotes from published authors. 